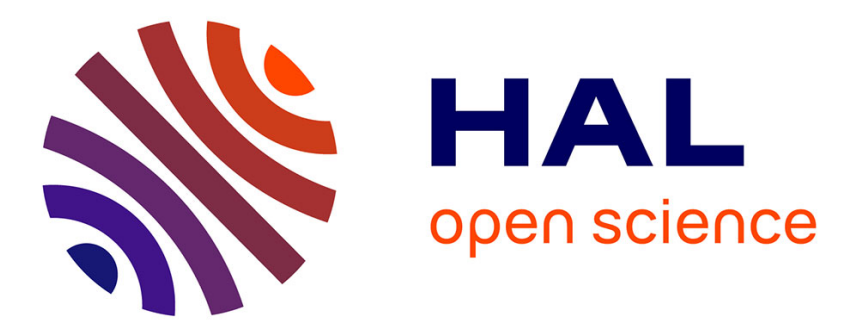

\title{
Geodesic network method for flows between two rough surfaces in contact
}

\author{
Franck Plouraboué, Frédérique Flukiger, Marc Prat, Pierre Crispel
}

\section{To cite this version:}

Franck Plouraboué, Frédérique Flukiger, Marc Prat, Pierre Crispel. Geodesic network method for flows between two rough surfaces in contact. Physical Review E: Statistical, Nonlinear, and Soft Matter Physics, 2006, vol. 73, pp. 036305-1. 10.1103/PhysRevE.73.036305 . hal-00959372

\section{HAL Id: hal-00959372 https://hal.science/hal-00959372}

Submitted on 14 Mar 2014

HAL is a multi-disciplinary open access archive for the deposit and dissemination of scientific research documents, whether they are published or not. The documents may come from teaching and research institutions in France or abroad, or from public or private research centers.
L'archive ouverte pluridisciplinaire HAL, est destinée au dépôt et à la diffusion de documents scientifiques de niveau recherche, publiés ou non, émanant des établissements d'enseignement et de recherche français ou étrangers, des laboratoires publics ou privés. 


\title{
Geodesic network method for flows between two rough surfaces in contact
}

\author{
F. Plouraboué, F. Flukiger, M. Prat, and P. Crispel \\ Institut de Mécanique des Fluides de Toulouse, UMR CNRS-INPT/UPS No. 5502 Avenue du Professeur Camille Soula, \\ 31400 Toulouse, France
}

\begin{abstract}
A discrete network method based on previous asymptotic analysis for computing fluid flows between confined rough surfaces is proposed. This random heterogeneous geodesic network method could be either applied to surfaces described by a continuous random field or finely discretized on a regular grid. This method tackles the difficult problem of fluid transport between rough surfaces in close contact. We describe the principle of the method as well as detail its numerical implementation and performances. Macroscopic conductances are computed and analyzed far from the geometrical percolation threshold. Numerical results are successfully compared with the effective medium approximation, the application of which is also studied analytically.
\end{abstract}

\section{INTRODUCTION}

When two parallel solid surfaces are brought into close contact, it is generally impossible to achieve perfect contact. Surface roughness is generally the cause of this contact mismatch. The void between those solid surfaces permits the passage of some fluid (gas or liquid). Such an apparently insignificant phenomenon can have dramatic consequences in many practical applications. An important event which attracted the attention of a larger audience to tightness issues was the space shuttle Challenger accident in 1986. A scientific commission was set up by the U.S. president with the aim of elucidating the origin of this catastrophic event. The presidential commission headed by Richard Feynman [1] concluded that a leakage in a seal joint was responsible for the explosion. The space shuttle joints are generally not composed of the usual polymeric material because of their very high operating temperatures and pressure. Instead, metallic solid surfaces are mutually compressed to prevent leakage [2]. This is nevertheless a difficult technological problem since a very large number of such seal gaskets with different mechanical properties are used. Similar sealings problems arise in other technical contexts such as nuclear plants, thermal exchange systems, and seal process technology in spatial satellites.

Mechanical engineering and hydrogeology are other contexts where fluid flows in between solid surfaces in contact. In the former, the metal-forming process generally involves lubricated close contacts between two solid surfaces. The cold-rolling process is an example where the fluid flow though "plateaus" necessitates knowledge of the microhydrodynamics between two surfaces near the geometrical percolation threshold [3].

In hydrogeology, flows though fractures play important roles in many rocks of low permeability [4,5]-e.g., in deep fractured reservoirs where the influence of normal constraints affects the permeability of fractures. In many cases, bulk normal constraints lead to a large number of contact regions between the solid surfaces, so that the fracture effective permeability depends drastically on the contact area.

As complicated as the problem appears, it can nevertheless be quite simplified if we recall that, in those contexts, the fluid transport is only coupled with the solid's mechanical deformation through the aperture field deformations of the solid surfaces. Usually no back-coupling of the flow pressure on the solid deformation has to be considered when the solid surfaces are rocks (except in the case of hydrofracturing processes) or metal surfaces. The latter can thus be used as input for studying the fluid flow inside the joint as in [6]. The computation of the fluid flow in such a highly complicated geometry is nevertheless a challenging problem. Direct numerical simulations of three-dimensional NavierStokes equations in complicated random geometries are very limited $[7,8]$. In fact, the closer to the geometrical percolation threshold we work, the finer the mesh must be [5]. Hence, a direct numerical simulation of the flow on a large percolating random field is definitively out of reach of any present or even future computer, as will be more precisely discussed in Sec. II B. The method we put forward tackles this problem by using previous results [9]. This previous analysis has permitted a mapping between the transport problems between continuous random surfaces and a discrete network built by linking maxima of the aperture field. These maxima are "pores" through which the flow tries to find a path. The only possible path between the pores is across constrictions associated with saddle points of the aperture field. Similar discrete methods have a long-standing history in the porous media literature [10-13]. It should nevertheless be noted that the method proposed in this paper is not a model but the application of a rigorous asymptotic approximation of the complete continuous hydrodynamical problem. Other studies such as $[14,15]$ have also considered related potential models to tackle continuum percolation problems mapped onto discrete networks. Weinrib [14] was the first to propose that a mapping should be associated with the continuum potential critical points. His study was related to optical problems. It is important to note that such an approximate description should nevertheless be carefully adapted to the problem at hand when deciding which mapping is to be applied at the discrete network level. In this paper different transport problems such as electrical, thermal, or fluid flows confined between solid surfaces in close contact are considered and discussed. 
The paper is organized as follows. Section II introduces the context and the key steps in the discrete mapping method. The precise algorithmic implementation of the network geometrical construction is discussed in detail in Sec. III. Then, macroscopic conductance coefficients are computed and compared with the effective medium approximation (EMA) in Sec. IV.

\section{FROM CONTINUOUS TO DISCRETE GEOMETRY}

This section describes how a discrete mapping on a discrete network can be obtained from an asymptotic analysis of the continuous problem.

\section{A. Short correlated slowly varying surfaces}

In the following, we consider sufficiently regular random surfaces which are at least $\mathcal{C}^{2}$. This class of random surfaces may appear restrictive. Nevertheless, it should be borne in mind that physical multiscaled surface roughness is always limited within a finite range of scales. Hence, below the lower cutoff of a multiscale distribution, there should always be a scale for which the regularity property of the surface derivative is meaningful. Here, we will furthermore restrict our point of view to surfaces having finite correlation length. It should be stressed that although simple, this class of surface can already involve multiscaled structures when percolation transport problems are considered. As will be discussed in Sec. II B, the long-range correlation of the percolation cluster increasingly dominates the effective surface for transport as the percolation threshold gets closer. Hence, we will consider in the following evolving surfaces for which some short-range correlation random structure is already present far from the percolation threshold. Let us define the Cartesian coordinates in three dimensions by $\left(x_{1}, x_{2}, x_{3}\right)$.

Let us now consider a stationary random field $h\left(x_{1}, x_{2}\right)$ for which the two-point correlation function defined from the probabilistic averaged $\langle\cdots\rangle$,

$$
C\left(x_{1}, x_{2}\right)=\left\langle\left[h\left(x_{1}^{\prime}, x_{2}^{\prime}\right)-h\left(x_{1}^{\prime}-x_{1}, x_{2}^{\prime}-x_{2}\right)\right]^{2}\right\rangle,
$$

depends only on the relative position $\left(x_{1}, x_{2}\right)$ of the two points considered. Among stationary random fields, Markovian fields are those for which $C\left(x_{1}, x_{2}\right) \rightarrow 0$ at some distance $\left\|\left(x_{1}, x_{2}\right)\right\|$ greater than some given finite correlation length $\ell$, $\left\|\left(x_{1}, x_{2}\right)\right\| \gg \ell$. The method we present in this paper is suited to such two-dimensional continuous random fields. Such objects can be encountered in different problems where the continuous field $h\left(x_{1}, x_{2}\right)$ is the local aperture between two surfaces: first, in a fracture defined as the void space between two faces of a crack. Even if the cracks have been identified as long-range correlated surfaces (see, for example, [16]), a typical correlation length can be found in fractures because of the relative displacement between the two faces of the crack [17] or other physicochemical mechanisms [5]. Another example of an application where rough surfaces play an important role, already mentioned in the Introduction, is the static gasket for preventing leakage in mechanical engineering. In this domain again, many man-made rough sur- faces have been found to be multiscaled (see, for example, $[18,19])$ but with nevertheless well-defined upper and lower cutoffs. The upper cutoff of such multiscaled roughness defines the characteristic correlation length scale of the surface. The impact of the multiscale roughness distribution below this characteristic scale is expected to be small because the dominant length scale controls the aperture and thus the transport properties associated with flow between the two surfaces (see, for example, [17,20]). In the following, a single, typical length scale associated with a well-defined correlation length will be considered. Such random surfaces can be obtained from prescribing a specific Fourier spectrum $\left|\tilde{h}\left(k_{1}, k_{2}\right)\right|^{2}$ of the aperture field $h\left(x_{1}, x_{2}\right)$. In the following, we compute the transport properties of a short-correlated surface with isotropic Gaussian correlation, for which the power spectrum is also Gaussian:

$$
\left|\tilde{h}\left(k_{1}, k_{2}\right)\right|^{2}=A e^{-\ell^{2}\left(k_{1}^{2}+k_{2}^{2}\right)} .
$$

The amplitude parameter $A$ is directly related to the surface rms roughness. Let us now discuss some remarkable geometrical properties of slowly varying surfaces.

\section{B. Flow transport in between slowly varying random surfaces}

The purpose of this section is to discuss the interest of critical points of a slowly varying random surface in flow transport problems. As mentioned in the Introduction, this paper is concerned with transport properties of flows through rough surfaces. In this context, the interesting geometrical random field to consider is the local distance between two rough surfaces. Let us describe the upper and lower rough surfaces through their respective surface heights $z_{u}\left(x_{1}, x_{2}\right)$ and $z_{l}\left(x_{1}, x_{2}\right)$. For simplicity, $z_{l}\left(x_{1}, x_{2}\right)$ and $z_{u}\left(x_{1}, x_{2}\right)$ are centered so that their spatial average is zero-i.e., $\left\langle z_{u}\right\rangle=\left\langle z_{l}\right\rangle=0$. We will consider random surfaces with the same statistical properties as those previously discussed in Sec. II A. As in several previous works [5,21], the local aperture field $h_{a}\left(x_{1}, x_{2}\right)$ is then simply defined by

$$
\begin{gathered}
h\left(x_{1}, x_{2}\right)=z_{u}\left(x_{1}, x_{2}\right)-z_{l}\left(x_{1}, x_{2}\right)+H, \\
h_{a}\left(x_{1}, x_{2}\right)=Y\left[h\left(x_{1}, x_{2}\right)\right] h\left(x_{1}, x_{2}\right),
\end{gathered}
$$

where $Y$ is the Heaviside function and $H$ is a level-set criterion that controls the relative mating of the two surfaces. Relation (3) is a crude geometrical model for surface deformations due to normal stress compression. Equation (3) describes a purely plastic deformation and stands as a "firstorder" geometrical approximation of surface confinement. Once in contact, the upper and lower surfaces do not interpenetrate and do not deform. As mentioned in the Introduction, surface deformations are decoupled from transport properties. This is because we consider a complete decoupling between the hydrodynamic and elastic constraints in the solid surfaces. This hypothesis is consistent with a large number of applications, particularly for tightness problems. Hence the methodological aspects developed in this paper for computing transport properties are independent of the deformation model and should remain relevant when elastic 

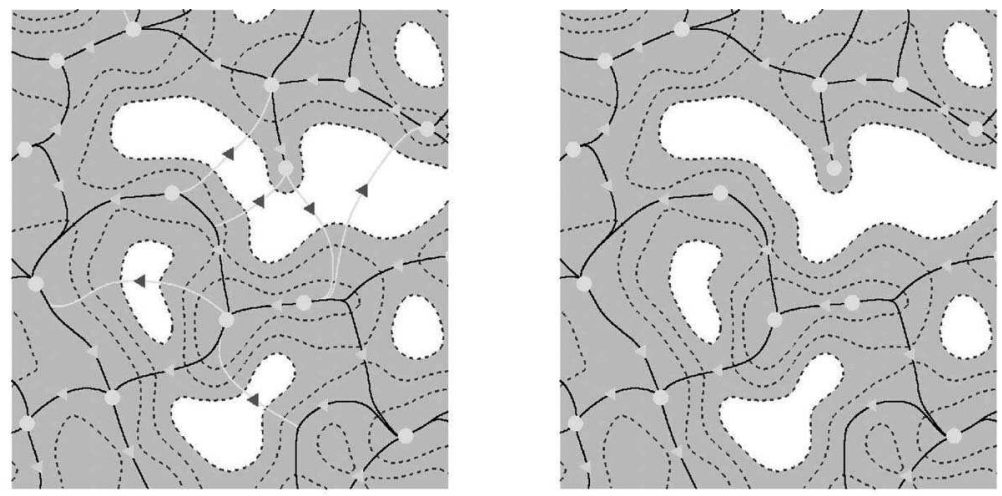

(a)

(b)

FIG. 1. Schematic representation of the geodesic network. White regions are associated with contacts regions between the two surfaces where the local aperture is zero, while grey regions are those associated with nonzero aperture regions. Some level sets of the aperture map are represented with dotted curves in those gray regions. The geodesic network linking maxima is represented with solid black lines, where the maxima are represented with grey solid circles. The saddle-point position along those geodesic network is represented with triangles. (a) All the links are represented. Active links are in black, and nonactive links are in grey. (b) Only the active links are represented.

deformation is included as studied in [6]. $H$ could be continuously varied when compressing one surface onto the other until the geometrical percolation threshold is reached. Hence, the closer to the percolation threshold, the more singular is the aperture field $h_{a}$.

It should be noted that the spatial variations $h_{a}\left(x_{1}, x_{2}\right)$ drastically control the flow path between two confined solid surfaces. Now it is easy to understand why we only consider the network of aperture maxima.

The only possible path between maxima crosses constrictions associated with saddle points. Hence the geodesic network presented in the previous section is the topological skeleton of continuous paths linking the pore network. This skeleton is illustrated in Figs. 1 and 2. Let us first qualitatively describe the evolution of the network structure when the surfaces are forced into close contact. To that purpose let us first define as "active" links [black lines in Fig. 1(b)] those associated with nonzero local aperture. Nonactive links are associated with contact regions where the local aperture is equal to zero [dotted lines in Fig. 1(a)]. Given the total number of links, $L_{t o t}$, in the absence of any contact, there is a smaller number of active links, $L<L_{c}$, in the network due to the contact region forced by nonzero values of the level set criterion $H$ in Eq. (3).

For a given critical $H_{c}$, the number of active links reaches a critical value $L_{c}$ associated with the geometrical percolation threshold from boundary $x_{2}=0$ to $x_{2}=2 \pi$. Below this critical value, there will be no percolating path from $x_{2}=0$ to $x_{2}=2 \pi$ on the graph network. Then, the usual parameter to consider in order to quantify the distance to percolation threshold is $p_{L}=\left(L-L_{c}\right) / L_{t o t}$. This parameter is the order parameter of the percolation transition. When $p_{L}=0$, the percolation threshold is reached. So the larger the values of $p_{L}$, the farther the geometrical percolation threshold. This could be observed in the network structure represented in Fig. 2. For values of $p_{L}$ as "large" as 0.25 the network structure looks reasonably homogeneous, while for values smaller than 0.1 large holes can be observed associated with increasing correlation length of the percolation cluster of the contact re- gions. It is interesting to note that the contact and noncontact regions are dual in two dimensions, so that the percolation threshold for noncontact regions is the same as the one for contact regions. An increasing correlation between contact regions thus has a direct impact on the induced increasing correlation of the active link network, which progressively smears out the preexisting finite correlation length $\ell$. This geometrical transition from a homogeneous to highly heterogeneous network has a direct impact on the transport properties. This paper further investigates the transition between the percolation regime and the breakdown of the mean-field approximation for transport coefficients in Sec. IV.

When considering large-scale transport properties one needs a large number of correlation lengths along with spatially averaged transport properties. It is then clear that the numerical representation of such continuous surfaces involves an even larger number of discrete elements. Computing transport properties over this finely discretized surface over many correlation lengths rapidly becomes impossible.

A previous analysis [9] has shown that the local aperture of each saddle point controls the spatial discretization necessary for computing the flow field. The lower the saddle-point aperture is, the smaller the spatial discretization should be. Hence tight constrictions associated with a very small saddle-point local aperture necessitate a challenging number $m$ of discretization points (as many as $m=512$ when using a finite-difference method of resolution for a ratio of aperture of the order of one-tenth between the saddle point and its adjacent maxima [9]). These considerations make it clear that the computational cost makes a direct computation of the flow unrealistic when the surface considered contains a large number of correlation lengths. There is nevertheless a way around this. A previous study [9] estimates the effective conductance of a continuous bond passing through a saddlepoint between two maxima of the aperture field. This estimation obtained with matched asymptotic expansion provides an analytical estimate for conductances as a function of the saddle-point local geometrical properties. This analysis then permits the mapping of three-dimensional continuous partial 


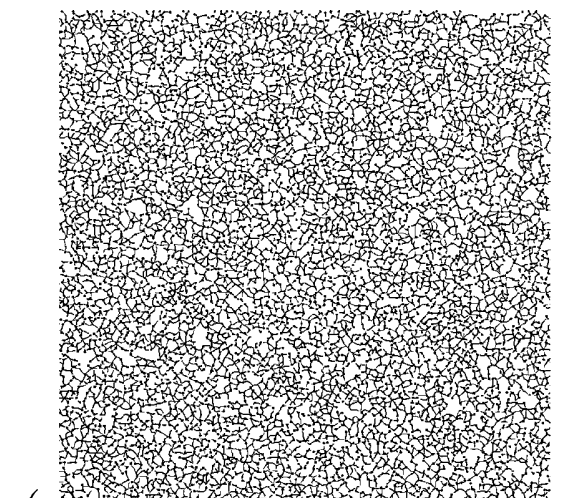

(a)

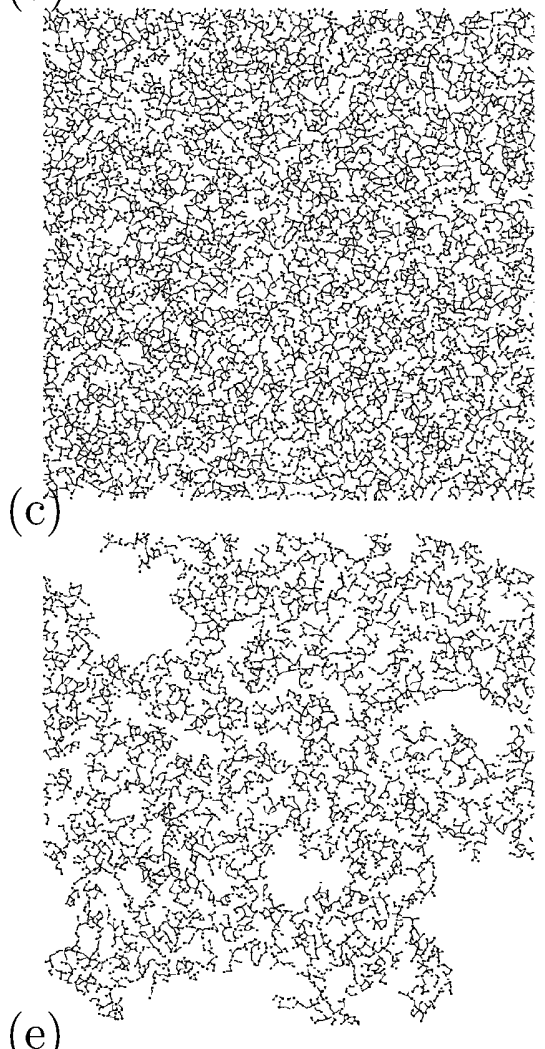

(b)

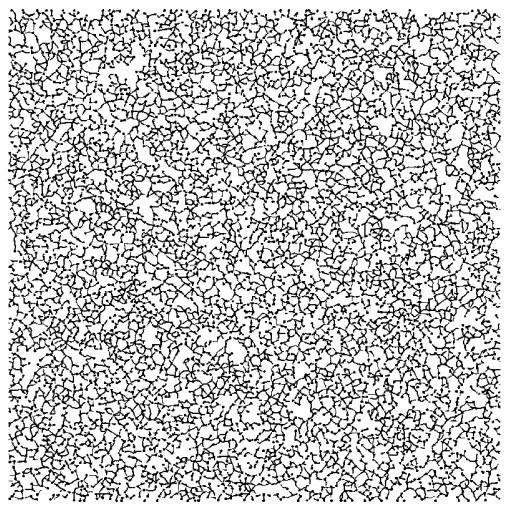

(d)
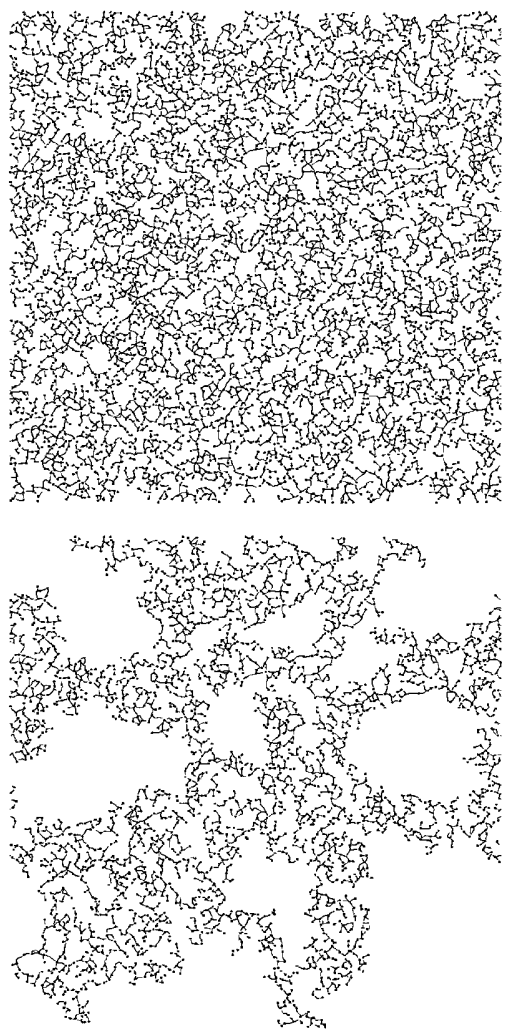

FIG. 2. Geodesic network for a $N^{2}=1024^{2}$ discrete surface, with $N / \ell=163$ for six different values of the level set $H$ of (3). Associated values of the parameter $p_{L}=\left(L-L_{c}\right) / L_{\text {tot }}$ are 0.45 in (a), 0.35 in (b), 0.25 in (c), 0.15 in (d), 0.1 in (e), and 0 in (f). The level-set values have been chosen so that (a) represents the original surface network with all its links, whereas (f) is the extreme case of the network at percolation threshold. The same conventions as Fig. 1 have been chosen to represent maxima with black circles, while the geodesic network is drawn with solid black lines.

differential problems (i.e., harmonic and Stokes problems) on a slowly varying spatial aperture field onto the discrete network of maxima linked with effective conductance bonds.

Here we discuss the scaling of the "discrete mapping" asymptotic results with the saddle-point geometrical properties. Let us consider the scalar field $\phi_{n}\left(x_{1}, x_{2}\right)$ associated with flow in between two rough surfaces. The scalar field $\phi_{n}$ could be a pressure $(n=3)$, an electrical potential $(n=1)$, or a temperature $(n=1)$.

The mapping problem then consists in finding the equivalent conductance $g_{n}$ that could account for the local potential loss $\Delta \phi_{n}$ between two connected maxima, given the total flux $\mathbf{q}$ that flows between those maxima. More precisely, the total flux $\mathbf{q}$ can be evaluated as the integral of the projection of the scalar field potential gradient $\nabla \phi_{n}$ (e.g., the pressure gradient for $n=3$ ) onto the normal to a surface defined at the saddle point. This surface intersects the saddle point located at $\left(x_{1 s}, x_{2 s}\right)$ which connects the two maxima. It is defined by its normal which is parallel to the eigenvector of the Hessian matrix whose eigenvalue $H_{+}\left(x_{1 s}, x_{2 s}\right)$ is positive. Then there is a linear relation between the flux and potential loss $\mathbf{q}=$ $-g_{n} \Delta \phi_{n} / \mu_{n}$, where $\mu_{n}$ is a physical parameter that depends on the problem under study; for $n=1$ it could either be the inverse of the fluid diffusivity or the inverse of the fluid electrical conductivity; for $n=3$ it is the dynamic viscosity of the fluid. The conductance coefficient $g_{n}$ is then related to the 
local geometrical properties of the saddle point by the following relation [the result (56) of Ref. [9] should have been written in its dimensional formulation]:

$$
g_{n}=c_{n} \sqrt{\frac{H_{+}\left(x_{1 s}, x_{2 s}\right)}{-H_{-}\left(x_{1 s}, x_{2 s}\right)}} h^{n}\left(x_{1 s}, x_{2 s}\right) \text {, }
$$

where $H_{+}\left(x_{1 s}, x_{2 s}\right)$ and $H_{-}\left(x_{1 s}, x_{2 s}\right)$ are the positive and negative Hessian eigenvalues at the saddle point and $c_{n}$ is a constant coefficient. The eigenvalues $H_{+}$and $H_{-}$are also the inverse of the positive and negative radii of curvature at the saddle point from relation (A5). In the following, all the surfaces considered are normalized with root-mean-square (rms) roughness $\sigma$ equal to 1 . This is equivalent to nondimensionalizing the local conductances $g_{n}$ by $\sigma^{n}$. Thus the results associated with the macroscopic conductances $G_{n}$ are also nondimensionalized by $\sigma^{n}$. The scaling obtained in relation (4) can be obtained with simple arguments. First, the dependence on the $n$th power of the local aperture field $h^{n}$ can be simply derived from the previously mentioned lubrication results that the local conductance already scales as $h^{n}$. The dependence on the square root of the radius of the curvature ratio also derives from this lubrication approximation property. It is worth noting that the effective conductance could be expressed as the composition of parallel resistance integrated along the flow trajectories. In the vicinity of the saddle point, these trajectories are locally parallel to the Hessian positive eigenvector direction. Let us call this direction $x_{+}$. Because the potential drop is mainly controlled by rapid variations localized in the vicinity of the saddle point, the total conductance along one trajectory can be approximated by the composition of conductances in series along $x_{+}$. Hence, integrating along all trajectories will introduce another composition of parallel conductances along the $x_{-}$direction. Hence a formal evaluation of $g_{n}$ without explicitly considering its prefactor is

$$
g_{n} \sim \int d x_{-} \frac{1}{\int d x_{+} \frac{1}{h^{n}\left(x_{+}, x_{-}\right)}}
$$

Now since, in the vicinity of the saddle point, the aperture field has a quadratic shape, it can be approximated by

$$
\begin{aligned}
h\left(x_{+}, x_{-}\right) \simeq & h\left(x_{1 s}, x_{2 s}\right)+\frac{1}{2} H_{+}\left(x_{1 s}, x_{2 s}\right) x_{+}^{2} \\
& +\frac{1}{2} H_{-}\left(x_{1 s}, x_{2 s}\right) x_{-}^{2}+\cdots .
\end{aligned}
$$

Rescaling $x_{+}$as $x_{+}^{\prime}=x_{+} / \sqrt{H_{+}}$and $x_{-}$as $x_{-}^{\prime}=x_{-} / \sqrt{-H_{-}}$then, one easily find from (5) and (6) that $g_{n} \sim \sqrt{-H_{+} / H_{-}}$. Hence each scaling obtained in Eq. (4) can be found from simple arguments. The computation of the prefactor $c_{n}$ is much more difficult and necessitates the use of matching asymptotic techniques. It was found in [9] that $c_{1}=2 / 3$ and $c_{3}=1 / 14$.

The asymptotic validity of (5) was checked numerically in [9]. To that purpose, the small parameter which is the ratio between the saddle-point aperture and the local radius of curvature at the saddle point was systematically varied. More precisely defining this small parameter as $\sqrt{h\left(x_{1 s}, x_{2 s}\right) H_{+}\left(x_{1 s}, x_{2 s}\right)}$, Eq. (4) is the lowest-order estimate of the conductance in the asymptotic expansion involving this small parameter.

Surprisingly, it was found in [9] that the validity range of the proposed asymptotic approximation was quite broad. Equation (4) gives a reasonable estimate (to within 20\%) of the conductance for values of the small parameter as large 3 in the case $n=3$. The case $n=1$ is less favorable; still, the value of the small parameter could be as large as 1 for a $20 \%$ estimate, as obtained in [9] from numerical computation.

We have now described how the mapping of continuous transport problems on critical points of the aperture field could be obtained. Let us now discuss how the discrete mapping is related to geometrical properties of slowly varying random surfaces.

\section{Principles of the geodesic network method}

To map the continuous transport problem onto a discrete network, it is first interesting to consider the critical points of the surfaces. For any of these critical points, one can easily retrieve maxima, minima, and saddle points. Each of these is found from the property of the local Hessian matrix $\mathbf{H}_{i j}$ $=\partial_{i j}^{2} h$ at each critical point. The saddle points are those where the determinant of the Hessian matrix is negative: det $\mathbf{H}$ $<0$. Maxima are the points where det $\mathbf{H}>0$ and the trace of the Hessian matrix is negative, $\operatorname{Tr} \mathbf{H}<0$, while minima are those for which det $\mathbf{H}>0$ and $\operatorname{Tr} \mathbf{H}>0$. Among these extrema, saddle points play a particular role in the flow transport as discussed in Sec. III B. From the geometrical point of view, saddle points are interesting for they are points which connect the shortest paths along the surface linking one maximum to another. Starting from one extremum to reach another one, the surface geodesics thus cross one saddle point. Hence, this property also occurs for the shortest paths linking minima which are orthogonal to geodesics of the maxima at each saddle point. We will not consider the minima's geodesic in the following, for reasons that will become obvious in the next section. We will rather concentrate on the construction of the geodesic network of the maxima. Two maxima are considered "connected" if a geodesic links them to the same saddle point. Figure 1 illustrates this connection between maxima through saddle points on a spatially slowly varying surface. Geodesics are simpler to compute on slowly varying surfaces than in the general case for they coincide with steepest descent or steepest ascent path as shown in the Appendix. We now briefly discuss the numerical implementation of this discrete mapping method.

\section{NUMERICAL IMPLEMENTATION}

This section focuses on the numerical implementation of the method whose theoretical basis was discussed in the previous section. Different algorithmic choices are possible. We tried to find a compromise between computational efficiency, robustness, and simplicity. The implementation of the method for the computation of macroscopic transport conductances follows different steps. 
First critical points need to be found. From these, the maximum geodesic network must be computed in order to find the bounds between maxima. Boundary conditions are then applied to this biperiodic two-dimensional network. For that purpose, maxima linked with each boundary should be identified. Then, for each level set $H$ controlling the aperture field (3) one needs to extract the percolating connected component of the network discrete graph. The part of the graph which is disconnected from the percolating component does not contribute to the flow transport. It is thus discarded. We describe each of these steps in the following. Before giving a more detailed description of the algorithmic implementation, let us first add some important remarks. Numerically, one has to discretize the field $h\left(x_{1}, x_{2}\right)$ obtained from computing the inverse Fourier transform of $\tilde{h}\left(k_{1}, k_{2}\right)$ defined in (2) on a $[0,2 \pi] \times[0,2 \pi]$ domain. Then we choose a ratio between the typical correlation length $\ell$ and the chosen discretization. The robustness of the following algorithm drastically depends on this discretization and on the precision required to define saddle points. Some numerical estimates indicate that a spatial discretization larger than six points per correlation length in the spectral representation gives a $10^{-8}$ relative error on the critical point positions. This level of discretization is implicitly considered in the following, while not being restrictive. Some of the algorithmic parameters have nevertheless to be adapted to this arbitrary discretization. Let us now discuss the different steps in more detail.

\section{A. Aperture field generation and critical point determination}

We use relation (2) to numerically generate surfaces as described in [22]. The prescription of a suitable Hermitic discretized complex field $\tilde{h}(i, j)$ with $i=1, N_{1}, j=1, N_{2}$ in Fourier space imposes a real discretized biperiodic field $h(i, j)$ in direct space.

Then, the discrete field $h(i, j)$ being defined, it is a classical and nontrivial algorithmic problem to extract its critical points. We chose a multigrid algorithm coupled with a classical Newton-Picard method to identify critical points. First, fast-Fourier-transform techniques were used to compute the vector gradient $\boldsymbol{\nabla} h(i, j)$ and Hessian matrix $\mathbf{H}_{i, j}$ fields of the discrete random field $h(i, j)$ on the Cartesian equispaced grid $(i, j)$. When needed on arbitrary points $\left(x_{1}, x_{2}\right)$ in the plane, any field can be interpolated with an arbitrary polynomial precision. We set quadratic interpolation degrees by default. Higher-order contributions of the polynomial order could be increased if the Newton method research failed after some arbitrary number of steps (which is chosen equal to 80). After convergence, the polynomial interpolation could also be increased during a new search if the required precision is not reached. Nevertheless, in view of the first discretization level, the required precision is always obtained with low interpolation order. The total number of extrema, $n_{e}$, is directly proportional to the chosen discretization, $n_{e} \propto N_{1} N_{2}$, up to a prefactor that depends on the chosen correlation length $\ell$. The ratio $N_{1} N_{2} / n_{e}$ is exactly equal to $m^{2}$, the gain on discretization when the network is used instead of the original surface. This ratio depends on the original discretization level as well as on the correlation length. For reasonable discretization levels, it reaches two orders of magnitude. It will be shown in the following that this is of considerable importance for the flow computation.

\section{B. Connecting critical points}

Critical point characteristics are easily obtained from the determinant and trace of the Hessian matrix $\mathbf{H}$, as already mentioned in Sec. II C. The connection between maxima of the field $h$ is obtained from the computation of geodesics starting from each saddle point. This computation is achieved with a steepest ascent method starting from each saddle point. The convergence criterion to this maximum is the same as the one used with the Newton search for critical points. Nevertheless, when the step size becomes too small, the algorithm switches from the steepest ascent method to the previous Newton search. This method provides very good results for linking saddle points to their corresponding maxima. The maximum-saddle-maximum triplet will be called a link in the following. Considering the list of all maxima, the computation of a new link connection will require a search over the whole list of maxima. The cost of this search can be greatly reduced by using a bucket sort to structure the list of maxima into a coarse bucket grid.

\section{From continuous to discrete boundary conditions}

Once the network is built, one has to map the continuous problem boundary conditions onto the discrete network. Below, Dirichlet and periodic boundary conditions are considered. The $x_{1}$ direction has periodic boundary conditions, while constant Dirichlet potential boundary conditions are applied along $x_{2}$ at $x_{2}=0$ and $x_{2}=2 \pi$. The network constructed using the previously described method is biperiodic. It thus necessitates some modifications to take into account the Dirichlet boundary condition of the potential in the $x_{2}$ direction. Each periodized link crossing the lines $x_{2}=0$ and $x_{2}=2 \pi$ has to be considered. For each of these crossing links, we need to find the "inside" and "outside" maxima. This is done by considering that the outside maximum is the one for which the $x_{2}$ coordinate needs to be periodized. At this point, the inside maximum is assigned to the boundary under consideration (i.e., $x_{2}=0$ or $x_{2}=2 \pi$ ) while the outside maximum is assigned to the other boundary (i.e., $x_{2}=2 \pi$ or $x_{2}=0$ ). Hence, for each link crossing the boundary, two new links are created, and the crossing link is suppressed from the link list.

\section{Graph operations on percolating clusters}

The subsections above have discussed the network construction. This section gives a detailed presentation of the operation needed on the discrete graph network to be able to compute the flow transport onto the topological skeleton of the aperture network. These procedures are related to the topology of the graph, while approaching the percolation threshold.

One first needs to extract the connected component of the graph network. This is achieved with a deep search algorithm 
implemented following classical methods [23].

The second step of graph operations is related to the identification of percolating and nonpercolating connected components. The geometrical percolation criterion of the topological skeleton is slightly different from the continuous aperture field. In the latter, the geometrical percolation is defined by finding any continuous path from the $x_{2}=0$ to the $x_{2}=2 \pi$ boundaries. When a discrete representation $h(i, j)$ of the aperture field is considered, this percolation criterion is defined on site percolation along nonzero values of the Cartesian grid $h(i, j)$. Now, transposing the geometrical percolation criterion onto the topological skeleton, one needs to consider bound percolation along the graph links which permits a path from $x_{2}=0$ to $x_{2}=2 \pi$ to be found inside the graph. This procedure is applied for each imposed level-set criterion $H$ until no path can be found for a threshold value $H_{c}$. A dichotomy iterative procedure on the level-set value $H$ is used to find the critical $H_{c}$. Once again, a deep search algorithm was implemented to achieve this purpose.

Since they do not contribute to the transport properties, the dead ends of each percolating connected component could have been suppressed. This task is nevertheless unnecessary because dead ends do not represent a large proportion of the network links; nor do they complicate the flow transport problem. On the contrary, the suppression of dead ends from the graph is technically involved (see, for example, [24]) and rather expensive algorithmically.

\section{E. Solving the linear system for the potential computation}

In Sec. III D we extracted the connected components of percolating clusters. The links that are related to boundaries $x_{2}=0$ and $x_{2}=2 \pi$ are also known from Sec. III C, as a subclass of the percolating components sets. This subclass has now to be extracted from the link sets so that Dirichlet boundary conditions can be applied. More precisely the potential $\phi_{n}$ has to be imposed so that $\phi_{n}\left(x_{1}, 0\right)=0$ and $\phi_{n}\left(x_{1}, 2 \pi\right)=1$. The corresponding set of boundary links can then be used to construct the right-hand side of the linear system for computing the potential. On the left-hand side, the rigidity matrix is built up from the sets of interior links using a classical Kirchoff conservation law at each node [25]. The use of compact storage for the memory allocation of the sparse rigidity matrix makes for very low memory costs for solving the linear system. A generalized minimal residual method preconditioned by a partial LU decomposition was chosen for the inversion of the linear system. This iterative method has been compared with a direct method near the percolation threshold. The comparison is useful, because the rigidity matrix is extremely ill conditioned, and it might happen that the convergence accuracy could be poor. It turns out that we did not find out any difference in the results up to the computer's double-precision digits.

These numerical procedures allow the computation of the hydraulic conductances of joints having a large number $N$ of correlation lengths in each $(x, y)$ direction. The computational cost of the procedure has been drastically reduced so that many statistical averages can be performed over the obtained results on a monoprocessor PC. The following section
TABLE I. Averaged coordinance number $z$ versus the total number of correlation length along each direction $x_{1}$ and $x_{2}$ of the surface. The averaged ratio of total link number at the percolation threshold $L_{c}$ over the total number of links $L_{t o t}$ is also given.

\begin{tabular}{|c|c|c|c|c|}
\hline$N / \ell$ & 40.7 & 81.5 & 163 & 326 \\
\hline$z$ & 3.63 & 3.80 & 3.89 & 3.94 \\
\hline$Z=z / 2-1$ & 0.82 & 0.90 & 0.95 & 0.97 \\
\hline$L_{c} / L_{t o t}$ & 0.5177 & 0.5110 & 0.5046 & 0.5011 \\
\hline
\end{tabular}

illustrates some of the numerical results obtained and their analysis, far from the percolation threshold.

\section{ANALYSIS OF TRANSPORT PROPERTIES FAR FROM THE PERCOLATION THRESHOLD}

This section investigates the macroscopic conductances by the numerical method described in the previous sections. We first describe the effective medium approximation and its use for computing macroscopic conductances. We then discuss the comparison between this approximation and direct numerical computations. We finally consider an analytical approximation of the EMA approach for the hydraulic effective conductance $G_{3}$.

\section{A. Mean-field EMA}

The EMA literature for scalar problems in heterogeneous media is very rich. We refer to [26] for a good recent overview of this topic. Our interest in the present paper is to apply such a method to the problem of conductances between two surfaces. As will be seen, this is an original problem for which new results can be established. The main point is to find good approximations for the effective macroscopic conductances, which will be denoted $G_{n}$ in the following with $n=1$ or $n=3$. These macroscopic effective conductances are associated with the discrete bound network of aperture maxima described in the previous section. Along this discrete bound network, local conductances are defined through (4). Hence, one can compute the set of all local conductances $\left\{g_{n}\right\}$, averaged over different realizations, with the associated probability density function (PDF) $p\left(g_{n}\right)$. Given the network mean coordinance number $z$, the EMA then proposes an integral equation formulation for the effective macroscopic conductances $G_{n}$ :

$$
\int_{0}^{\infty} \frac{g_{n}-G_{n}}{g_{n}+Z G_{n}} p\left(g_{n}\right) d g_{n}=0
$$

where the coefficient $Z$ is related to the averaged coordinance number $z$ by the simple relation $Z=z / 2-1$. This formula is written in the limit of an infinite aperture field $h\left(x_{1}, x_{2}\right)$. Hence, the value of $Z$ should be evaluated on an infinite surface. This is obviously not possible in practice because physical objects have a finite number of correlation lengths. A good approximation is thus to compute $z$ for a large but finite number of correlation lengths. Numerical estimates are given the values in Table I. 


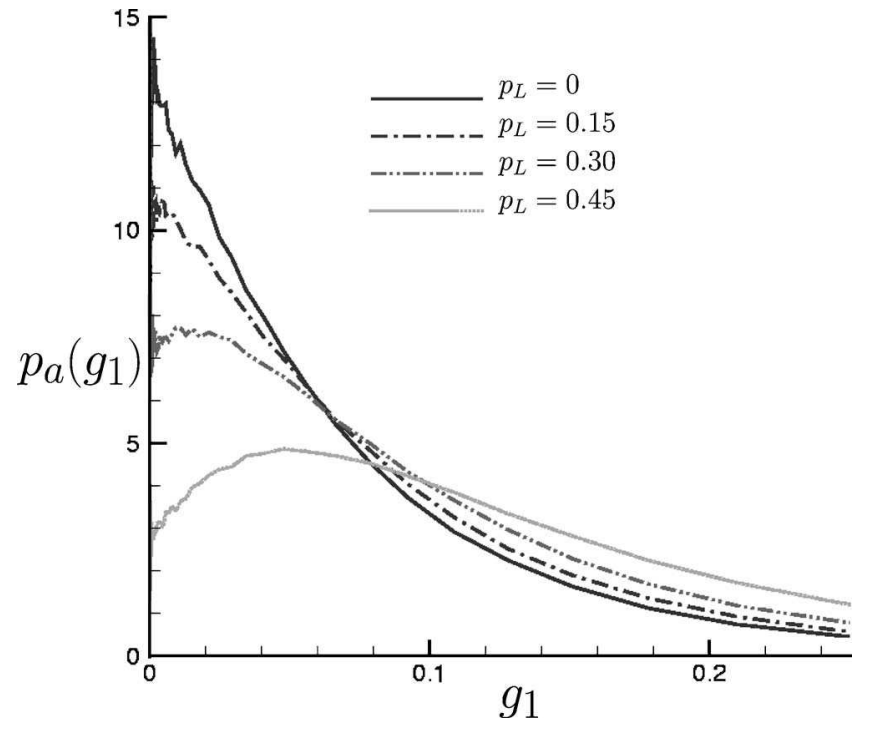

FIG. 3. PDF of the local conductances $p_{a}\left(g_{1}\right)$ computed numerically for different values of the link percolation threshold $p_{L}=(L$ $\left.-L_{c}\right) / L_{\text {tot }}$.

The statistical fluctuations associated with the averaged results of Table I are lower than the given number of digits. The PDF $p\left(g_{n}\right)$ distribution associated with the local conductances describes both "active" links [black lines on Fig. $1(b)$, for which the local aperture is nonzero, and "nonactive" ones associated with contact regions with zero local aperture [grey lines in Fig. 1(a)]. Given the total number of links, $L_{t o t}$, and the total number of active links, $L$, the PDF can be decomposed, so that

$$
p\left(g_{n}\right)=\left(1-F_{a}\right) \delta(0)+F_{a} p_{a}\left(g_{n}\right),
$$

where $F_{a}=L / L_{t o t}$ denotes the fraction of active links whose PDF distribution is $p_{a}\left(g_{n}\right)$. Using Eq. (8) to describe the relation between the macroscopic conductance and the PDF distribution of active links in Eq. (7) it is easy to find that

$$
\int_{0}^{\infty} \frac{g_{n}-G_{n}}{g_{n}+Z G_{n}} p_{a}\left(g_{n}\right) d g_{n}=\frac{\left(1-F_{a}\right)}{Z F_{a}} \text {. }
$$

Far from the percolation threshold, this relation should give a good approximation of the macroscopic conductance. It is therefore an interesting approximation to compare with direct numerical computations obtained from the method described in the previous section. This method nevertheless requires knowledge of the active link PDF $p_{a}\left(g_{n}\right)$. This PDF can be computed numerically over a large number of local conductances obtained from relation (4). Let us first discuss the numerical evaluation of EMA macroscopic conductances.

\section{B. Numerical evaluation of the EMA and comparison with numerical computations}

The results of the numerical computation of the local conductance PDF distribution $p_{a}\left(g_{1}\right)$ associated with the case $n$ $=1$ is represented in Fig. 3. As the level set $H$ is increased, the active link number $L$ is decreased and the control parameter $p_{L}=\left(L-L_{c}\right) / L_{\text {tot }}$ tends to 0 . The smaller $p_{L}$, the more the local conductance distribution peaks near zero. This is obvious since the number of local conductances near the contact regions is maximal at the percolation threshold. Nevertheless, there is no divergence of the PDF as $g_{1} \rightarrow 0^{+}$. On the contrary, when the PDF distribution $p_{a}\left(g_{3}\right)$ associated with the case $n=3$ represented in Fig. 6(a), is below, considered the local hydraulic conductance distribution diverges when approaching zero. A bilogarithmic representation of this PDF shows that this divergence is algebraic, with a well-defined exponent over more than four decades. There is apparently a very weak dependence of this algebraic behavior on the control parameter $p_{L}$, while a secondary peak in the distribution nevertheless appears far from the percolation threshold. This power law distribution can be understood from relation (4). The local conductance is related to the local aperture $h$ to the power $n$. The Hessian eigenvalue ratio modifies this conductance value but should always remains of order 1 [9]. Moreover, the Hessian eigenvalue ratio does not change when surfaces are brought into contact using model (3). Hence the main features of the conductance PDF are due to its $h^{n}$ dependence. We have empirically verified that suppressing the Hessian eigenvalue ratio term in the computation of the local conductances affects neither its PDF nor the EMA computation for the effective conductances. Hence, in the following, we will rather concentrate on the dependence of the conductance PDF on the local aperture term. From (4), the relation

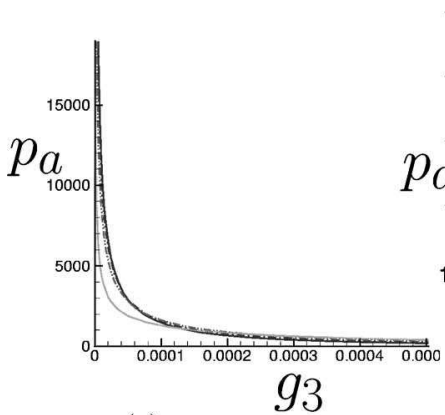

(a)

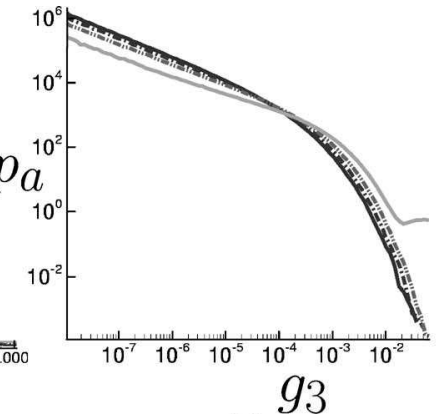

(b)

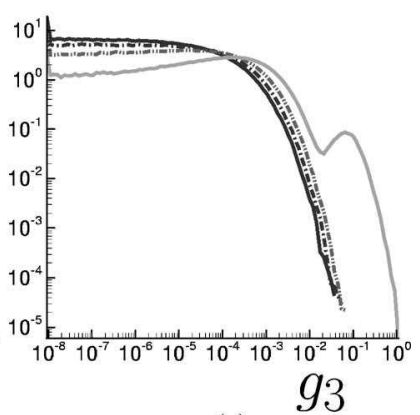

(c)

FIG. 4. PDF of the local conductances $p_{a}\left(g_{3}\right)$ computed numerically for different values of the link percolation threshold $p_{L}=(L$ $\left.-L_{c}\right) / L_{\text {tot }}$. Same conventions as Fig. 3 for different values of $p_{L}=0$ : solid black curve; 0.15 , dash-dotted back curve; 0.3 , dash-dotted gray curve; 0.45 , solid gray curve (a) usual coordinates, (b) bilogarithmic representation, and (c) bilogarithmic representation of $p_{a}\left(g_{3}\right) g_{3}^{2 / 3}$ versus $g_{3}$. 

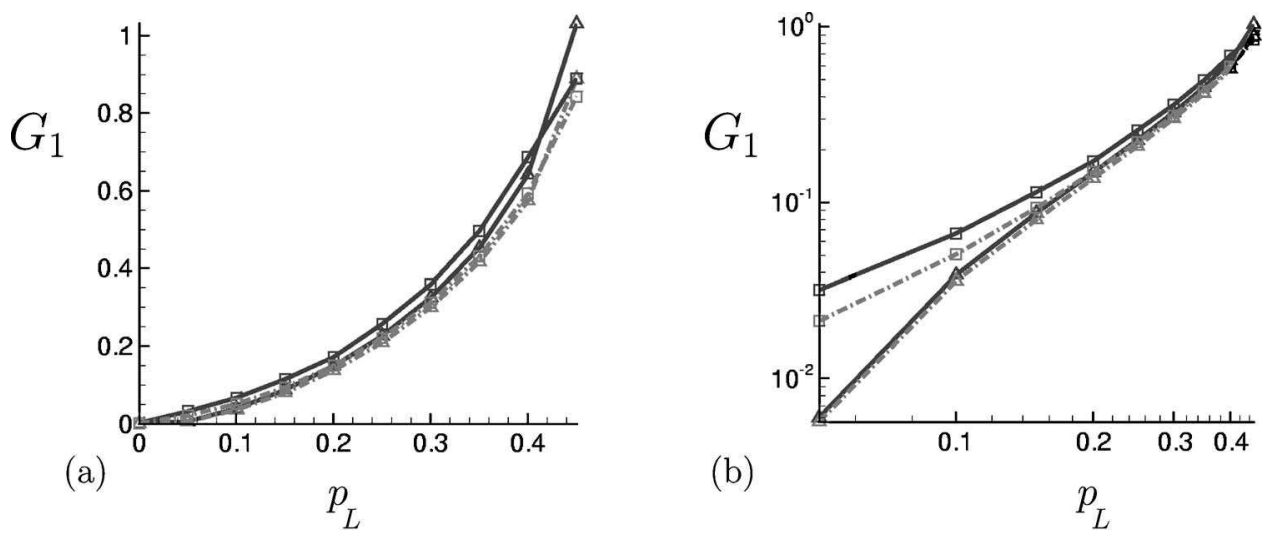

FIG. 5. Effective conductance $G_{1}$ computed numerically for different values of the link's percolation threshold $p_{L}=\left(L-L_{c}\right) / L_{t o t}$. Finitesize effects are tested either on direct numerical computation or on EMA computation. Hence, solid lines refer to $N / \ell=40.5$ while dot-dashed lines refer to $N / \ell=81.5$, the number of correlation lengths. Triangular symbols refers to the EMA while square symbols are associated with direct computation. (a) Usual coordinates and (b) bilogarithmic representation,

between the conductance PDF and the local aperture PDF is found to be $p_{a}\left(g_{n}\right) \sim p_{h}(h)\left(d h / d g_{n}\right)$, so that

$$
p_{a}\left(g_{n}\right) \sim g_{n}^{-(n-1) / n} p_{h}\left(g_{n}^{1 / n}\right) .
$$

Hence, in the case $n=1$, there is no singular behavior of the PDF conductance as $g_{1} \rightarrow 0^{+}$, while in the case $n=3$, the local conductance diverges algebraically such that $p_{a}\left(g_{3}\right) \sim g_{3}^{-2 / 3}$. This property is closely related to the one examined in [15], for which the local conductance has a power law divergence as $g_{n} \rightarrow 0$. This scaling is investigated in the PDF histograms in Fig. 4(c). Figure 4(c) confirms that the scaling is relevant for a large range of decades over the smallest values of the local conductances. Hence a reasonable approximation for the hydraulic conductance PDF over a wide range of scales should be written in the simple form

$$
p_{a}\left(g_{3}\right) \simeq \mathrm{Cg}_{3}^{-2 / 3} .
$$

The constant $C$ depends on the control parameter $p_{L}$-i.e., the distance to the percolation threshold. As expected, numerical computations showed a linear relation between $C$ and $p_{L}$. The coefficients of these linear relations are slightly dependent on the system size. In order to further investigate this observation, the number of correlation lengths in each direction of the system was varied from $N / \ell=81.5$ to $N / \ell$ $=326$ to look for changes in the local distributions as shown in Figs. 3 and 4 for which $N / \ell=81.5$. Very few differences were noticed. In both $n=1$ and $n=3$ cases, each PDF conductance preserved the same generic properties. The impact of finite-size numerical effects on the numerical computation only slightly affected the coefficients of the linear relation $C\left(P_{L}\right)$. In the following we will denote this linear relation by

$$
C\left(P_{L}\right)=a P_{L}+b
$$

The numerical evaluation of those coefficients gives $a$ $=6.32$ and $b=-9.06$.

The numerical computation of conductances PDF $p_{a}\left(g_{n}\right)$ allowed the macroscopic conductances $G_{n}$ to be computed by applying relation (9) for different values of the control parameter $P_{L}$. This computation was carried out using a simple trapezoidal rule for the integration. Different histogram binnings were tested to investigate the impact of the numerical integration on the result. The relative error obtained was below $1 \%$. These results were compared with direct numerical computation using the method described in Sec. III. The re-
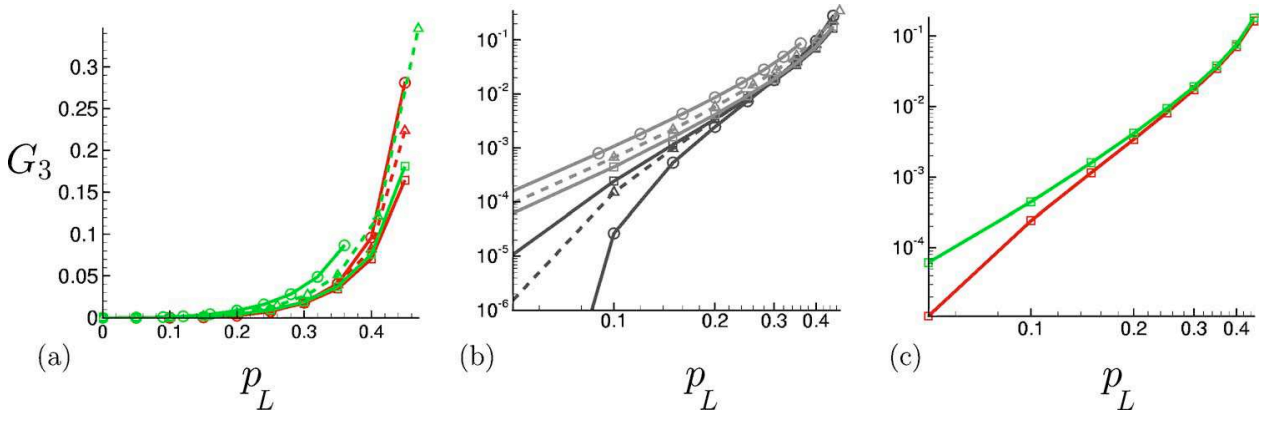

FIG. 6. (Color online) Effective hydraulic conductance $G_{3}$ computed numerically for different values of the link percolation threshold $p_{L}=\left(L-L_{c}\right) / L_{t o t}$. Finite-size effects are investigated either with direct numerical computation (grey curves) or EMA computation (black curves). Solid lines with square symbols refer to $N / \ell=163$ and $N=1024$ while dashed lines with triangular symbols refer to $N / \ell=81.5$ and $N=512$ and dotted lines with circle symbols to $N / \ell=40.7$ and $N=256$. (a) Usual coordinates, (b) bilogarithmic representation, and (c) bilogarithmic representation for only $N / \ell=163$ and $N=1024$. 


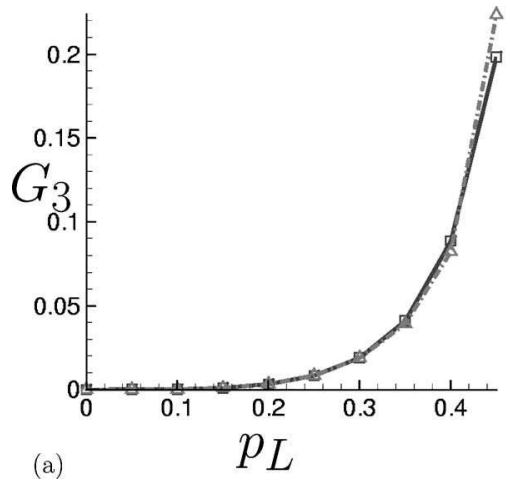

(a)

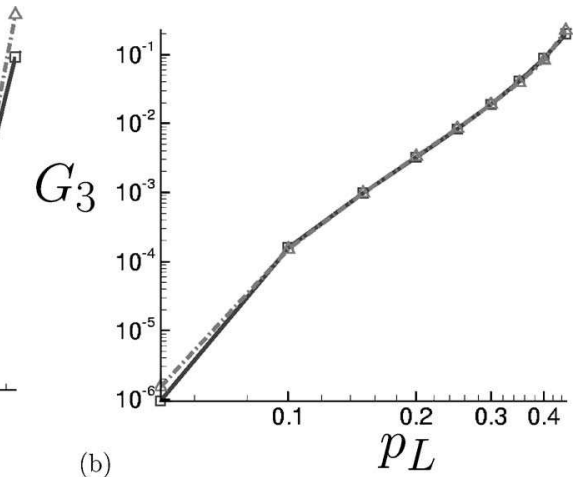

FIG. 7. Comparison between the hydraulic effective conductances $G_{3}$ versus $p_{L}=\left(L-L_{c}\right) / L_{\text {tot }}$ obtained from EMA numerical computation (dotdashed line) and analytical expression (16) (square symbol linked with the solid line). (a) Usual coordinates and (b) bilogarithmic representation. sults of the direct numerical computation were averaged over a large number of realizations, the number of which nevertheless depends on the system size $N$ (typically from a thousand for $N=256$ to a few tens for $N=2048$ ). The size of the points represented in Figs. 5-7 were chosen larger than the error bars coming from the statistical fluctuations associated with the average.

The comparison showed a very good agreement between both computations far from the percolation threshold. In the case $n=1$ illustrated in Fig. 5(a), the comparison indicates that the EMA gives better than $15 \%$ precision in the effective conductance far from the percolation threshold. It is also interesting to examine where the breakdown of the EMA arises in the vicinity of the percolation threshold. It can be observed in Fig. 5(b) that this breakdown occurs for parameter values $P_{L}$ smaller than 0.1 in the case $n=1$. Finite-size effects are moreover observed in this case when changing the number of correlation lengths in each direction from $N / \ell$ $=40.7$ to $N / \ell=81.5$. Nevertheless, the impact of finite-size effects does not affect the good comparison between direct numerical computation and EMA results far from the percolation threshold. In both cases, it can be seen in Fig. 5(b) that the breakdown of the EMA occurs for the same value $P_{L}$ $=0.1$. This difference between EMA and direct simulations far from the percolation threshold drops down to $5 \%$ in the case $n=3$ as illustrated in Fig. 6(a). Finite-size effects are nevertheless more important, as illustrated in Figs. 6(a)-6(c). Far from percolation threshold, the influence of finite-size effects is illustrated in Fig. 6(a). Although the difference between direct numerical simulation and EMA drops to $5 \%$ for $N / \ell=163$ correlation lengths, it can rise to $30 \%$ for $N / \ell$ $=81.5$. The impact of finite-size effects on the breakdown criteria of the EMA can also be observed in Fig. 6(c), where the critical value for $P_{L}$ varies from 0.05 to 0.1 when $N / \ell$ varies from 81.5 to 163 . These results indicate that the EMA gives a good approximation over quite a large range of area surface fractions of contact, in the limit of a large number of correlation lengths in the system.

Let us now find an analytical expression for approximating the effective conductances.

\section{Analytical approximation of the EMA}

In this subsection we seek an analytical expression for the effective hydraulic conductance obtained from the EMA. It would be interesting to find an analytical estimate in order to propose a general expression that could be more easily used on related problems. Such an analytical approximation is not possible in the $n=1$ case. In this case, the integral formulation (9) does not admit any asymptotic approximation, since it is not dominated by the simple behavior of the local conductance PDF $p\left(g_{1}\right)$. More precisely, the integral (9) diverges logarithmically with its lower bound. An asymptotic expression of the integral as a function of the lower bound can be found but the contribution of the upper bound cannot be neglected. It turns out that no closed form can be found for $G_{1}$ as a function of the lower and upper bounds in that case.

In contrast, in the case $n=3$, the simple behavior of the local conductance PDF $p\left(g_{3}\right)$ expressed in relation (11) dominates the left-hand-side integral of relation (9). The lower and upper integral bounds should nevertheless be equal to 0 and $1 /(3 C)^{3}$, so that PDF (11) is normalized on this interval. Using those integral bounds and (11) in the left-hand-side integral of relation (9) leads to an implicit and cumbersome relation that relates the effective conductances $G_{3}$ to $C, Z$ and obviously $F_{a}=L / L_{t o t}$ :

$$
\begin{aligned}
\frac{3 X\left(1 / F_{a}-1-Z\right)}{(1+Z)}= & \frac{1}{2} \ln \left(1-\frac{3 X}{(1+X)^{2}}\right)-\sqrt{3} \arctan \left(\frac{2 X-1}{\sqrt{3}}\right) \\
& -\frac{\pi \sqrt{3}}{6},
\end{aligned}
$$

where $1 / X=3 C\left(Z G_{3}\right)^{1 / 3}$. This relation can be further simplified by using the fact that the effective conductance $G_{3}$ is always quite small-i.e., $G_{3} \ll 1$ - whatever value of the control parameter $F_{a}=L / L_{t o t} \leqslant 1$ is. Thus, we seek an asymptotic expansion of relation (13) as $X \gg 1$. Keeping with $O\left(1 / X^{2}\right)$ and discarding $O\left(1 / X^{4}\right)$, the right-hand side of Eq. (13) can be expanded to give

$$
\begin{aligned}
\frac{3 X\left(1 / F_{a}-1-Z\right)}{(1+Z)}= & \frac{1}{2}\left(-\frac{3}{X}+\frac{3}{2 X^{2}}\right)-\sqrt{3}\left(\frac{\pi}{2}-\frac{\sqrt{3}}{2 X}-\frac{\sqrt{3}}{4 X^{2}}\right) \\
& -\frac{\pi \sqrt{3}}{6} .
\end{aligned}
$$

From Eq. (14), it can be seen that the root of the following cubic equation gives an approximate expression for the effective conductance: 


$$
X^{3} \frac{3\left(1 / F_{a}-1-Z\right)}{1+Z}+X^{2} \frac{2 \pi}{\sqrt{3}}-\frac{3}{2}=0 .
$$

An explicit solution for $X$ and thus for $G_{3}$ can be obtained by computing the smallest positive root of this cubic equation. This leads to the following explicit analytical expression:

$$
\begin{aligned}
& G_{3}=-\frac{81 \sqrt{3}\left(1 / F_{a}-1-Z\right)^{3}}{8 \pi^{3} C^{3}(1+Z)^{3} Z[1+\cos (\theta / 3)+\sin (\theta / 3)]^{3}}, \\
& G_{3}=-\frac{81 \sqrt{3}\left[1 /\left(P_{L}+L_{c} / L_{t o t}\right)-1-Z\right]^{3}}{8 \pi^{3} C^{3}(1+Z)^{3} Z[1+\cos (\theta / 3)+\sin (\theta / 3)]^{3}},
\end{aligned}
$$

where $\theta=\arccos \left[\left(2187 \sqrt{3} / 8 z^{2} \pi^{3}\right)\left(1 / F_{a}-1-Z\right)^{2}-1\right]$. Moreover, the constant $L_{c} / L_{\text {tot }}$ reaches an asymptotic value of $1 / 2$ (see Table I) for a system of infinite size and the constant $C$ is given in Eq. (12). The comparison of this analytical expression with the numerical evaluation of the EMA obtained in the previous section is displayed in Fig. 7 with respect to the control parameter $P_{L}$. The maximal relative error found by varying the control parameter $P_{L}$ from $10^{-1}$ to 0.45 is smaller than $2 \%$. Hence, Eq. (16) is a useful expression to consider for approximating the EMA results.

\section{CONCLUSION}

This paper has proposed a method for computing fluid flows in between random rough surfaces in contact. The method is based on mapping the continuous transport equations onto the discrete network of the aperture's maxima previously proposed in [9]. This mapping allows the local discrete conductances between maxima to be computed as a function of the saddle-point geometrical properties. A numerical implementation of this method has been proposed.

The computation gain for computing the effective macroscopic conductances turns out to be of major importance. The efficiency of this random heterogeneous geodesic network method has allowed the computation of the averaged macroscopic conductances associated with thermal, electrical $n=1$, or fluid $n=3$ transport. In every case, the macroscopic results have been compared with the mean-field EMA method. Far from the percolation threshold, the comparison turns out to be very good, leading to a $15 \%$ difference for $n=1$ to as low as $5 \%$ for $n=3$. This method thus enables a thoughtful analysis of the transport properties of tightly mated surfaces. It would be useful to analyze the transport properties near the geometrical percolation threshold.

\section{ACKNOWLEDGEMENTS}

We would like to thank Sylvain Mazoyer and Pierre Gomord for their help in the algorithmic implementation. This work has been supported by G.D.R 2345 "Etanchéité statique par joint métallique en conditions extrèmes" with financial help from CNRS, EDF, CNES, and SNECMA as well as the "Charles De Gaulle" foundation.

\section{APPENDIX: GEODESIC NETWORK OF CRITICAL POINTS ON SLOWLY VARYING ROUGH SURFACES}

This appendix shows how the curvature tensor defined on every point of a smooth $\mathcal{C}^{2}$ surface can be approximated by the Hessian matrix. From this approximation, it is shown how to construct the geodesic network linking all critical points by following steepest descent path along the surface.

Smooth short-correlated surfaces have well-defined critical points where the surface height gradient vanishes. More precisely let us first briefly discuss the properties of surfaces having slow variations in the $\left(x_{1}, x_{2}\right)$ horizontal directions associated with a small parameter $\epsilon$. Any point defined by its vector position $\mathbf{x}=\left(x_{1}, x_{2}, x_{3}\right)$ in three dimensions and located on a slowly varying surface $S$ defined as $x_{3}=h\left(\epsilon x_{1}, \epsilon x_{2}\right)$ has coordinates $\mathbf{x}=\left(x_{1}, x_{2}, h\right)$. Critical points are those for which

$$
\left(\frac{\partial h}{\partial x_{1}}, \frac{\partial h}{\partial x_{2}}\right)=(0,0) \text {. }
$$

It is now interesting to introduce slowly varying coordinates $\left(X_{1}=\epsilon x_{1}, X_{2}=\epsilon x_{2}\right)$ so that the surface variations are now expressed directly by $x_{3}=h\left(X_{1}, X_{2}\right)$. Let us denote the partial derivative $\partial / \partial X_{i}$ with respect to the slow Cartesian coordinate $X_{i}, \partial_{i}$, with $i=1,2$, and let us define $\nabla h=\left(\partial_{1} h, \partial_{2} h\right)$. Condition (A1) can now be expressed by

$$
\nabla h=\left(\partial_{1} h, \partial_{2} h\right)=\frac{1}{\epsilon}\left(\frac{\partial h}{\partial x_{1}}, \frac{\partial h}{\partial x_{2}}\right)=(0,0) .
$$

It is easy to see that, for a slowly varying surface, the local curvature tensor coincides with the surface Hessian. Let us define the tangent vector to the surface by $\left[\partial_{1} \mathbf{x} \equiv \mathbf{x}_{1}\right.$ $\left.=\left(1,0, \epsilon \partial_{1} h\right), \quad \partial_{2} \mathbf{x} \equiv \mathbf{x}_{2}=\left(0,1, \epsilon \partial_{2} h\right)\right]$. The outward-oriented normal $\mathbf{n}$ to the surface then reads

$$
\begin{gathered}
\mathbf{n}=\frac{\mathbf{x}_{1} \times \mathbf{x}_{2}}{\left\|\mathbf{x}_{1} \times \mathbf{x}_{2}\right\|}=\frac{1}{\sqrt{1+\epsilon^{2} \boldsymbol{\nabla} h^{2}}}\left(\epsilon \partial_{1} h, \epsilon \partial_{2} h, 1\right), \\
\mathbf{n}=\left(\epsilon \partial_{1} h, \epsilon \partial_{2} h, 1\right)+O\left(\epsilon^{2}\right),
\end{gathered}
$$

where $\times$ denotes the vector product between two vectors in three dimensions. The curvature tensor $\mathbf{b}$ is then defined in its two-covariant form as (see, for example, [27])

$$
\mathbf{b}_{i j}=-\mathbf{x}_{i} \cdot \mathbf{n}_{j} \quad \text { with } i=(1,2), \quad j=(1,2),
$$

where denotes the scalar product between two vectors in three dimensions and $\mathbf{n}_{j}$ is $\partial \mathbf{n} / \partial X_{j}, j=1,2$. Using (A3) the curvature tensor given in (A4) can easily be computed up to $O\left(\epsilon^{2}\right)$ terms as

$$
\mathbf{b}_{i j}=-\epsilon \partial_{i j}^{2} h=-\epsilon \mathbf{H}_{i j} .
$$

Hence, in this case, the principal curvature directions associated with the eigenvectors of the curvature tensor are always locally tangent to the Hessian eigenvectors. It is now easy to realise that, self-consistently, the steepest ascent path from one saddle point to its adjacent maximum will follow a geodesic of the smooth surface. The trajectory of any point along the surface is parametrized by time $t$. The steepest 
ascent path along the surface is defined for any surface points by the local variation $d\left(x_{1}, x_{2}\right) / d t=\epsilon \boldsymbol{\nabla} h$. The parameter $t$ can be prescribed to be equal to the third coordinate $x_{3}$ along the surface. Steepest ascent trajectories thus satisfy $d \mathbf{x} / d t$ $=(\epsilon \boldsymbol{\nabla} h, 1)$ in three dimensions, which from (A3), leads to $d \mathbf{x} / d t=\mathbf{n}$ neglecting $O\left(\epsilon^{2}\right)$ terms. Hence the tangent kinematic vector along the steepest ascent trajectory is always parallel to the surface normal. This is precisely the definition of a geodesic. Hence, steepest ascent trajectories are surface geodesics on slowly varying smooth surfaces. This property is used in Sec. III B to compute the geodesic network linking all maxima of the surface.
[1] W. P. Rogers et al., The Presidential Commission on the Space Shuttle Challenger Accident Report, Tech. rep., NASA (June 6, 1986).

[2] J. Martin (unpublished).

[3] N. Marsault, P. Montmitonnet, P. Deneuville, and P. Gratacos (unpublished).

[4] K. J. Evans, T. Kohl, R. J. Hopkirk, and L. Rybach (unpublished).

[5] P. Adler and J. Thovert, Fractures and Fracture Networks (Kluwer Academic, Amsterdam, 1999).

[6] V. V. Mourzenko, O. Galamay, J. F. Thovert, and P. M. Adler, Phys. Rev. E 56, 3167 (1997).

[7] D. Lo Jacono, F. Plouraboué, and A. Bergeon, Phys. Fluids 17, 063602 (2005).

[8] V. V. Mourzenko, J. F. Thovert, and P. M. Adler, J. Phys. II 5, 465 (1995)

[9] F. Plouraboué, S. Geoffroy, and M. Prat, Phys. Fluids 16, 615 (2004).

[10] M. B. Isichenko, Rev. Mod. Phys. 64, 961 (1992).

[11] J. Koplik, Disorder and Mixing (Kluwer, Dordrecht, 1988), pp. 123-137.

[12] M. Sahimi, Rev. Mod. Phys. 65, 1393 (1993).

[13] M. Sahimi, Flow and Transport in Porous Media and Fractured Rock (VCH Wienheim, New York, 1995).

[14] A. Weinrib, Phys. Rev. B 26, 1352 (1982).
[15] S. Feng, B. I. Halperin, and P. N. Sen, Phys. Rev. B 35, 197 (1987).

[16] S. R. Brown and C. H. Scholz, J. Geophys. Res. 90, 12 (1985).

[17] F. Plouraboué, P. Kurowski, J. P. Hulin, S. Roux, and J. Schmittbuhl, Phys. Rev. E 51, 1675 (1995).

[18] A. Majumdar and B. Bhushan, ASME J. Tribol. 112, 205 (1990).

[19] F. Plouraboué and M. Boehm, Tribol. Int. 32, 45 (1999).

[20] F. Plouraboué, P. Kurowski, J. Boffa, J. P. Hulin, and S. Roux, J. Contam. Hydrol. 46, 295 (2000).

[21] M. Prat, N. Letalleur, and F. Plouraboué, Transp. Porous Media 48, 291 (2002).

[22] H. A. Makse, S. Havlin, M. Schwartz, and H. E. Stanley, Phys. Rev. E 53, 5445 (1996).

[23] R. Tarjan, Data Structures and Network Algorithms (Society for Industrial and Applied Mathematics, New York, 1983).

[24] H. J. Herrmann, D. C. Hong, and H. E. Stanley, J. Phys. A 17, L261 (1984).

[25] S. V. Patankar, Numerical Heat Transfer and Fluid Flow (Hemisphere, New York, 1980).

[26] J. Torquato, Random Heterogeneous Material (Springer-Verlag, Berlin, 2001).

[27] T. Frankel, The Geometry of Physics: An Introduction (Cambridge University Press, Cambridge, England, 1997). 